\title{
IMPORTÂNCIA DA VITAMINA D EM COVID - 19
}

\author{
Anielly Nobrega D'agostino Sales ${ }^{1}$
}

\section{IINTRODUÇÃO}

A vitamina $D$ é um hormônio esteroide, produzido endogenamente com o efeito da radiação ultravioleta na pele ou disponível em fontes de alimentos exógenos ou suplementos dietéticos. A insuficiência de vitamina $\mathrm{D}$ é um problema de saúde pública que afeta mais de um bilhão de pessoas em todos os estágios da vida em todo o mundo (Holick, 2017).

$\mathrm{Na}$ última década, vários estudos demonstraram uma ligação potencial entre a deficiência de vitamina D e várias doenças, incluindo infecção sistêmica (Dankers, Colin, van Hamburg, Lubberts, 2017). A insuficiência de vitamina D afeta as funções imunológicas, pois a vitamina D exerce papel de imunomodulação (Bouillon et al., 2019), aumentando a imunidade inata pela secreção de peptídeos antivirais, o que melhora as defesas da mucosa. infecções do trato respiratório incluindo gripe epidêmica (Zhou, Luo, Qin, 2019).

Uma meta-análise recente incorporando dados de oito estudos observacionais relatou que indivíduos com concentração sérica de vitamina $\mathrm{D}<50 \mathrm{nmol} / 1$ (ou seja, <20 ng / ml) tiveram um risco 64\% maior de pneumonia adquirida na comunidade. Algumas revisões recentes hipotetizaram que a insuficiência de vitamina $\mathrm{D}$ pode comprometer a função imune respiratória, aumentando o risco de gravidade e mortalidade de COVID-I9 (Grant et. al, 2020).

\footnotetext{
${ }^{1}$ Doutoranda em Naturopatia Clínica. Pós graduanda em adequação nutricional e manutenção da homeostase - Dr Lair Ribeiro. Naturopata. Terapeuta ortomolecular. Hidrocolonterapeuta. Ozonioterapeuta Detoxterapeuta. Dermaticista. Pós- graduada em Medicina Espostiva. Graduada em Educação física. E-mail: mi_resolve@outlook.com
} 
Existem também alguns estudos retrospectivos que determinaram a correlação dos níveis de vitamina D com a gravidade e mortalidade do COVID-I9 (Daneshkhah et al., 2020). O surto e a rápida disseminação do SARS-CoV-2 são uma ameaça à saúde global com um desfecho instável em todo o mundo. Um dado recente relatou os efeitos antivirais da vitamina $\mathrm{D}$, que podem impedir a replicação viral diretamente e também ser eficaz de forma anti-inflamatória e imunomoduladora (Teymoori-Rad, Shokri, Salimi, Marashi, 2019).

Parece que a SARS-CoV-2 usa principalmente o processo de evasão imune durante a infecção, que é seguido por hiper reação e tempestade de citocinas em alguns pacientes, como um conhecido processo patogênico de desenvolvimento da síndrome de doenças respiratórias agudas (SDRA).

O SARS-CoV-2 usa a enzima conversora de angiotensina 2 como receptor hospedeiro para entrar nas células epiteliais alveolares e intestinais. A desregulação subsequente do sistema terenina-angiotensina pode levar ao excesso de produção de citocinas, resultando em SDRA fatal prospectiva (Jakovac, 2020).

Considerando as diferenças na gravidade e fatalidade de COVID-ı9 no globo, é importante entender as razões por trás disso. A melhora da imunidade por meio de uma melhor nutrição pode ser um fator considerável. O nutriente como a vitamina $\mathrm{D}$ mostra papéis significativos na função imunológica. No entanto, pouco se sabe sobre o papel da vitamina $\mathrm{D}$ na prevenção da infecção e da fatalidade por COVID-19. Um estudo avaliou a correlação das concentrações de vitamina $\mathrm{D}$ com casos e mortes de COVID-I9 por um milhão da população em 20 países europeus usando dados do portal de dados de pandemia COVID-19 (Tian, Rong, 2020) até 20 de maio de 2020 (a maioria dos países após o primeiro pico).

Esta revisão também discutiu o possível papel preventivo da vitamina $\mathrm{D}$ nas infecções agudas do trato respiratório. Além disso, foram discutidos os estudos disponíveis que determinaram o papel da vitamina $\mathrm{D}$ na gravidade e mortalidade da COVID-I9. 


\section{IVITAMINA D E COVID-19}

\section{I VITAMINA D E MECANISMOS PARA DIMINUIR INFECÇÕES VIRAIS}

Algumas revisões recentes demonstraram alguns caminhos pelos quais a vitamina $D$ diminui o risco de infecções microbianas. A vitamina $D$ segue diferentes mecanismos na redução do risco de infecção viral e mortalidade. Para reduzir o risco de resfriado comum, a vitamina $\mathrm{D}$ usa três vias: barreira física, imunidade natural celular e imunidade adaptativa (Gombart, Pierre, Maggini, 2020).

Uma revisão recente também apoiou o possível papel da vitamina $\mathrm{D}$ na redução do risco de infecções e mortalidade por COVID-ı. Compreende a manutenção de junções celulares e junções comunicantes, aumentando a imunidade celular diminuindo a tempestade de citocinas com influência no interferon, fator de necrose tumoral e regulando a imunidade adaptativa através da inibição das respostas de células $\mathrm{T}$ auxiliares tipo I e estimulação da indução de células T (Tian, Rong, 2020).

A suplementação de vitamina $\mathrm{D}$ também foi encontrada para aumentar a contagem de células $\mathrm{T} \mathrm{CD}_{4}$ + na infecção por HIV. Uma das principais manifestações de infecção grave por SARS-CoV-2 é a linfopenia.

Em ambos os modelos de camundongos e em linhas de células humanas, a vitamina $\mathrm{D}$ exerceu atividade no tecido pulmonar e teve efeitos protetores na pneumonite intersticial experimental.

Vários estudos in vitro demonstraram que a vitamina $\mathrm{D}$ desempenha um papel significativo na "homeostase respiratória" local, seja por estimular a exposição de peptídeos antimicrobianos ou por interferir diretamente na aplicação de vírus respiratórios.

A insuficiência de vitamina D pode, portanto, estar envolvida na SDRA e na insuficiência cardíaca e essas são as manifestações de indivíduos com COVID-ı9 gravemente enfermos. Portanto, a deficiência de vitamina D promove o sistema renina- 
angiotensina (SRA), que pode levar à doença cardiovascular crônica (DCV) e redução da função pulmonar.

Pessoas com essas comorbidades são responsáveis por uma porcentagem maior de casos graves de doença em COVID-19. Embora, muitos estudos apoiem as características imunomoduladoras da vitamina $\mathrm{D}$ e seu papel significativo na manutenção da homeostase imunológica; experimentos controlados randomizados bem desenhados são necessários para elucidar o papel plausível da vitamina $\mathrm{D}$ na resposta imune protetora contra micróbios respiratórios e na prevenção de vários tipos de infecções agudas do trato respiratório (Tsujino.2019).

\subsection{RELEVÂNCIA DA VITAMINA D PARA COVID-I9}

É importante elucidar completamente os mecanismos de virulência de COVID-I9, vários mecanismos celulares, incluindo a replicação mediada por protease semelhante à papaína (PLpro), ligação do receptor de dipeptidil peptidase-4 (DPP-4/ CD26), interrupção da proteína $M$ mediada indução de IFN tipo I e evasão de reconhecimento de hospedeiro MDA5 e RIG-I foram reconhecidos no COVID-MERS vírus intimamente relacionado (McCartney, Byrne, 2020).

DPP-4 / CD26 humano foi mostrado para se conectar com o domínio Si do pico de COVID-ı glicoproteína, sugerindo que também poderia ser uma infecção de fator de virulência saliente em Covid-19. A expressão do receptor DPP-4 / CD26 é reduzida significativamente in vivo após a correção da insuficiência de vitamina D (Vankadari, Wilce, 2020).

Há também uma indicação de que a manutenção da vitamina $\mathrm{D}$ pode reduzir algumas das sequelas imunológicas a jusante desfavoráveis que se acredita extrair um resultado clínico mais pobre na infecção por Covid-ı, como elevação de interleucina 6, resposta tardia de interferon-gama (Zdrenghea et al., 2017) e um prognóstico negativo marcador em indivíduos com pneumonia aguda, incluindo aqueles com Covid-r9.

Alguns estudos clínicos e epidemiológicos apoiam a hipótese sobre COVID-19 e sua relação com o estado de vitamina D. Estudos recentes indicaram que COVID-ig está 
associado ao aumento da geração de citocinas pró-inflamatórias, proteína $C$ reativa (PCR), SDRA, pneumonia e insuficiência cardíaca (Ali, 2020).

$\mathrm{Na}$ China, as taxas de mortalidade crônica foram de 6 a Io\% para pessoas com doenças crônicas do trato respiratório, doenças cardiovasculares, hipertensão e diabetes (Surveillances, 2020). Em outros estudos, as concentrações séricas de $25(\mathrm{OH}) \mathrm{D}$ foram inversamente associadas às citocinas pró-inflamatórias, IL-6, aumento da PCR e aumento do risco de pneumonia, SDRA, diabetes e insuficiência cardíaca (Pittas et al., 2019).

Em ensaios clínicos randomizados, a suplementação de vitamina D demonstrou reduzir o risco de doenças respiratórias. Um ensaio controlado por placebo com 5660 indivíduos mostrou que a suplementação de vitamina $\mathrm{D}$ reduz significativamente o risco de infecções do trato respiratório.

Uma revisão que incluiu cinco estudos clínicos relatou que as infecções do trato respiratório foram significativamente mais baixas no grupo com suplementação de vita mina $\mathrm{D}$ do que no grupo de controle (Hou, Zhao, Liu, 2019). Outro estudo incluiu 25 ensaios clínicos randomizados, com 10.933 participantes no total de I4 países diferentes indicaram os efeitos benéficos da suplementação de vitamina $\mathrm{D}$ na redução do risco de pelo menos uma infecção aguda do trato respiratório (Lips et al., 2019).

Dados sobre o número de casos e mortes de COVID-i9 por milhão da população em 20 países europeus foram obtidos em 20 de maio de 2020 (todos os países após o primeiro pico) (Tian, Rong, 2020).

Os dados para as concentrações médias de vitamina $\mathrm{D}$ sérica desses 20 países foram obtidos de uma publicação anterior (Lips et al., 2019). No teste de coeficiente de correlação de Pearson, uma correlação significativa $(r=-0,477, p=0,033)$ foi observada entre as concentrações médias de vitamina $\mathrm{D}$ e o número de casos COVID-19 / população de I $\mathrm{M}$, enquanto, a relação entre as concentrações médias de vitamina $\mathrm{D}$ e o número de mortes de COVID-ı9/ população de I M não foi significativa $(r=-0,357, p=0,123)$. Na presente análise, a correlação negativa entre os níveis de vitamina D e COVID -I9 casos é mais forte do que 
$(\mathrm{r}=-0,444, \mathrm{p}=0,050)$ observado em 8 de abril de 2018 (a maioria dos países antes do primeiro pico) em uma análise anterior (Ilie, Stefanescu, Smith, 2020).

No entanto, a correlação negativa da vitamina D com as mortes por COVID-ı9 na presente análise parece ser mais insignificante do que na análise anterior. O número de casos e mortes por milhão de populações variou de país para país nesses dois períodos de tempo.

Existe a probabilidade de que exista uma relação de causa e efeito. As diferenças subjacentes na distribuição de idade, composição populacional, condições médicas préexistentes e variação no uso de medicamentos podem ser variáveis potencialmente confusas que podem afetar a correlação. Outros estudos disponíveis sobre o papel da vitamina $\mathrm{D}$ em infecções e resultados por COVID-ıg Até agora, há uma falta de ensaios clínicos e estudos de coorte para determinar o papel preventivo da vitamina $\mathrm{D}$ nas infecções e na gravidade por COVID-19. No entanto, existem alguns estudos observacionais retrospectivos que determinaram a correlação entre os níveis de vitamina $\mathrm{D}$ e os casos e gravidade de COVIDI9, os resultados não foram consistentes para todos os estudos.

Por exemplo, um estudo retrospectivo usou um banco de dados de 212 pacientes com infecção de SARS-CoV-z e mediu os níveis séricos de vitamina $\mathrm{D}$ de três hospitais em países do sul da Ásia (Alipio, 2020). O autor observou uma diferença significativa nos níveis médios de vitamina D nos casos leve $(78 \mathrm{nmol} / \mathrm{L})$, comum $(68,5 \mathrm{nmol} / \mathrm{L})$, grave $(53 \mathrm{nmol}$ / L) e crítico de COVID-ig (p <o,oor ).

$\mathrm{O}$ autor observou uma associação significativa entre o estado de vitamina $\mathrm{D}$ e os resultados clínicos ( $\mathrm{p}<\mathrm{o}, \mathrm{oor})$. Em Cingapura, um pequeno estudo observacional de coorte incluiu 43 casos de um hospital acadêmico terciário relatou que menos pacientes com COVID-ıg que receberam a combinação oral doses de vitamina D (Iooo UI), $\mathrm{Mg}$ (I5o mg) e vitamina $\mathrm{B}_{12}$ (50o $\mathrm{mg}$ ) exigiram terapia subsequente dexigênio em comparação com os controles ( $3 / 17$ vs. $16 / 26, p=0,006)$. Foi observado que os pacientes tratados com combinação de vitamina $\mathrm{D}, \mathrm{Mg}$ e vitamina $\mathrm{B}_{12}$ mostraram efeitos protetores significativos contra a deterioração clínica $(\mathrm{p}=\mathrm{o}, \mathrm{o} 4 \mathrm{I})$, mesmo após ajuste para idade, sexo e comorbidades (Tan et al., 2020). 
Baixos níveis de vitamina $\mathrm{D}$ também foram relatados em pacientes graves com COVID-ı9 e pacientes com doenças pré-existentes (Glicio, 2020). Na Bélgica, um estudo observacional retrospectivo consistiu em 186 casos positivos e 2717 controles negativos, relatando uma mediana baixa significativa $(\mathrm{p}=0,0016)$ de vitamina $\mathrm{D}$ em pacientes com COVID-ı9 em comparação com os controles (De Smet et al., 2020).

Outro estudo usou conjuntos de dados de diferentes partes do mundo e demonstrou uma redução de I5\% no número de casos graves de COVID-ı devido a um status normal de vitamina $\mathrm{D}$ em uma população. Um estudo de coorte retrospectivo da Indonésia incluiu 780 casos relatados que casos mais velhos e homens com condições médicas pré-existentes e níveis de vitamina $\mathrm{D}$ abaixo do normal estão associados a maiores chances de morte (Raharusun et al., 2020).

Nesse estudo, após ajuste de fatores de confusão (idade, sexo e comorbidade), o status de vitamina D mostrou uma forte relação com a mortalidade por COVID-ı9 (Raharusun et al., 2020). Um estudo retrospectivo nos Estados Unidos que incluiu um grande número de casos demonstrou que a luz do sol e a vitamina $\mathrm{D}$, com latitude como um indicador, possivelmente estão associadas a riscos reduzidos para casos de COVID-I9 e mortalidade (Li et al., 2020).

Por outro lado, um estudo usou dados do UK Biobank (2006-2010) para o status da vitamina $\mathrm{D}$ e etnia e correlacionou com os casos de COVID-19 (Hastie et al., 2020); nenhuma diferença significativa foi observada para os níveis de vitamina $\mathrm{D}$ com casos de COVID-ı9 após o ajuste de potenciais fatores de confusão.

No entanto, a etnia mostrou uma associação significativa com a infecção por COVID-ıg na análise uni variada. Outro estudo usou os mesmos dados do UKBiobank (2006-2010) para IMC, status da vitamina D e etnia e observou alguns pontos interessantes (Darling et al., 2020).

Os autores não encontraram nenhuma diferença significativa para os níveis médios de vitamina $\mathrm{D}$ entre os casos COVID-ıg e o grupo controle. O nível de vitamina $\mathrm{D}$ foi significativamente mais baixo nas pessoas de etnia asiática, negra e mista ( $p<0,00 \mathrm{r})$ em 
comparação com as de etnia branca. Além disso, os níveis de vitamina $\mathrm{D}$ foram significativamente mais baixos naqueles com obesidade ( $p<0,00 \mathrm{r})$; indivíduos do sexo masculino com sobrepeso ou obesos que vivem em Londres e são de etnia asiática, negra ou mista foram associados a uma maior número de casos de teste positivos.

No entanto, no modelo de regressão, a interação entre o IMC e o status de vitamina D não prediz os resultados do teste no conjunto de dados disponível. É importante observar que existem alguns fatores potenciais associados à gravidade e mortalidade do COVID-I9, como idade, sexo, etnia , comorbidades e coinfecção. Um estudo determinou o impacto das comorbidades em 1.590 pacientes com COVID-I9 na China e relatou que os pacientes com COVID-I9 com qualquer comorbidade (hipertensão, diabetes) produziram resultados clínicos piores do que aqueles sem (Guan et al., 2020).

Outro estudo na Coreia do Sul indicou uma associação de comorbidades com infecção por COVID-ı9 e cursos clínicos graves (Ji et al., 2020). Uma revisão sistemática e meta-análise demonstraram que a idade e a doença subjacente, incluindo hipertensão, doença cardiovascular e doenças do sistema respiratório, são possíveis fatores de risco para pacientes graves com COVID-I9 em comparação com pacientes não graves (Yang et al., 2020).

Além das comorbidades, as coinfecções podem ser outro determinante importante de gravidade e mortalidade em pacientes com COVID-I9. As coinfecções bacterianas e fúngicas foram identificadas em pacientes com COVID-ı. Atualmente, não há evidências claras de que as pessoas que vivem com HIV corram um risco mais alto de infecção por COVID-ig.

No entanto, as pessoas que vivem com HIV e têm um sistema imunológico comprometido precisam de cuidado extra para prevenir infecções por COVID-I9.

A importância das coinfecções na gravidade das doenças respiratórias tem sido amplamente estudada; no entanto, eles são pouco estudados no surto de COVID-I9. Portanto, mais dados sobre coinfecções são necessários para determinar seus impactos na 
gravidade e mortalidade de COVID-19. Os efeitos benéficos da suplementação de vitamina D COVID-ı9 foi declarada uma pandemia global pela Organização Mundial de Saúde.

No entanto, as informações sobre os potenciais fatores de proteção dessa infecção são limitadas. Atualmente, não há evidências claras de que a suplementação de vitamina $\mathrm{D}$ previna a gravidade e a mortalidade da COVID-I9.

Existem alguns testes randomizados registrados na avaliação do papel da vitamina D nas infecções por COVID-ıg e severidades, mas ainda não relataram seus achados. Até agora, existe um pequeno estudo de coorte descrito acima (De Smet et al., 2020) que demonstrou os efeitos protetores da combinação de vitamina D, Mg e vitamina Bı2 contra a deterioração clínica de COVID-ı. Em uma meta-análise anterior, a suplementação de vitamina $\mathrm{D}$ foi mostrada como segura e eficaz na prevenção de infecções agudas do trato respiratório (Martineau et al., 2017).

Eles também acrescentaram que os indivíduos com deficiência grave de vitamina $D$ tiveram os benefícios máximos da suplementação. Os autores também notaram que o papel protetor da vitamina $\mathrm{D}$ era alto em indivíduos com níveis séricos de $25(\mathrm{OH}) \mathrm{D}$ basais $<25$ $\mathrm{nmol} / \mathrm{L}$ em comparação com aqueles com concentrações séricas de $25(\mathrm{OH}) \mathrm{D}>25 \mathrm{nmol} /$ L (Martineau et al., 2017).

No mesmo estudo, a análise de subgrupo indicou que a ingestão diária ou semanal de vitamina $\mathrm{D}$ (sem doses em bolus adicionais) mostrou efeitos protetores contra a infecção aguda do trato respiratório, especialmente em pessoas com deficiência de vitamina D. A suplementação com D também aumentou a expressão gênica relacionada à antioxidação (subunidade modificadora da glutationa redutase) (Lei, Zhang, Cheng, Lee, 2017).

O aumento da produção de glutationa poupa o uso de vitamina $C$, que tem potencial atividade antimicrobiana (Mousavi, Bereswill, Heimesaat, 2019), e tem sido sugerido para prevenir e tratar a infecção por COVID-ı. Até agora, há evidências limitadas de que a suplementação de vitamina $\mathrm{D}$ de 20-50 $\mu \mathrm{g} /$ dia tem algum efeito adverso à saúde.

De fato, a suplementação de vitamina D com doses de até ıoo $\mu \mathrm{g} / \mathrm{dia}$ é segura para adultos e muitos grupos de especialistas agora sugerem a suplementação em pessoas mais 
velhas, embora em níveis inferiores. Um estudo relatou que a ingestão de suplemento de vitamina $\mathrm{D}$ em Ioo-250 $\mu \mathrm{g} /$ dia durante 6 semanas aumenta a concentração sérica basal de $25(\mathrm{OH}) \mathrm{D}$ de 2 para 3 vezes, respectivamente, sem quaisquer efeitos adversos à saúde (Charoenngam et al., 2020).

Vale a pena sugerir tomar até $250 \mu \mathrm{g} /$ dia por um mês, o que é eficaz para aumentar os níveis séricos de $25(\mathrm{OH}) \mathrm{D}$ para a faixa ideal entre 75 e $125 \mathrm{nmol} / \mathrm{L}$. A dose pode ser reduzida para Ioo $\mu \mathrm{g} /$ dia após um mês para manter as concentrações circulantes de $25(\mathrm{OH})$ D (Charoenngam et al., 2020).

No entanto, em estudos clínicos futuros pode valer a pena avaliar a potência de diferentes planos de dosagem de vitamina $\mathrm{D}$ em infecções agudas do trato respiratório, por exemplo, uma vez por semana, o que pode ser mais fácil de implementar. e variáveis biológicas, como etnia, idade, duração da exposição, variações sazonais, índice de massa corporal, ingestão de certos medicamentos, concentração basal de vitamina $\mathrm{D}$, genética e tipos de suplementos de vitamina D (Fabbri, Infante, Ricordi, 2020).

Além disso, a heterogeneidade da população, bem como a dose de vitamina $\mathrm{D}$, precisa ser considerada durante a determinação do papel preventivo da vitamina D no COVID-I9. Para avaliar os impactos da suplementação de vitamina $\mathrm{D}$ em estudos randomizados controlados, deve-se dar ênfase ao desenho do estudo com base na concentração sérica de 25 $(\mathrm{OH}) \mathrm{D}$ em vez da concentração da dose administrada (Fabbri, Infante, Ricordi, 2020).

Uma revisão recente também sugeriu a suplementação de magnésio com suplementos de vitamina $\mathrm{D}$, pois o magnésio ajuda a regular a homeostase do fosfato e do cálcio (Grant et al., 2020).

As enzimas envolvidas no metabolismo da vitamina D parecem precisar de magnésio, que desempenha um papel importante como cofator nas reações enzimáticas, especialmente no rim e no fígado (Uwitonze, Razzaque, 2018).

Um número crescente de relatórios concordantes apoia um papel protetor da vitamina D na redução de pelo menos o risco / gravidade de infecções do trato respiratório 
(RTIs), especialmente no contexto de influenza e COVID-ig.(Munshi, Hussein, Toraih et al., 2020).

Os principais relatórios clínicos mostram que a deficiência de vitamina $\mathrm{D}$ contribui para a síndrome do desconforto respiratório agudo (SDRA) SARS-CoV-2 e que as taxas de letalidade aumentam com a idade e as concentrações séricas mais altas de SARS-CoV-2. (Vyas, Kurian, Bagchi et al, 2020).

Além disso, o surto de COVID-I9 parece ocorrer principalmente no inverno frio, quando as concentrações séricas de 25-hidroxivitamina D (25 (OH) D-calcidiol ou calcifediol) são as mais baixas, assim como as doses de ultravioleta B (UVB), enquanto o número de casos no hemisfério sul perto do final do verão é menor (Rhodes, Subramanian, Laird et al., 2020).

As medições direcionadas da concentração sérica de $25(\mathrm{OH}) \mathrm{D}$ e a suplementação de vitamina $\mathrm{D}$ são fortemente sugeridas como tendo importantes benefícios para a saúde pública e para o paciente (Grant, Al Anouti, Moukayed, 2020).

O papel positivo da terapia de reposição de vitamina D (vDRT) na redução do risco e da gravidade em pacientes com COVID-ıg é apoiado por várias evidências clínicas e RCTs estão sendo submetidos. No entanto, experiências anteriores de RCT relacionadas a vDRT estão disponíveis em outros estudos de infecção viral pulmonar e até mesmo em pacientes em unidade de terapia intensiva (UTI) para adultos ventilados mecanicamente (Grant, Lahore, McDonnell, et al., 2020).

Essas observações importantes são corroboradas por vários mecanismos biológicos / moleculares por meio da vitamina $\mathrm{D}$, que geralmente podem reduzir o risco de infecções e diminuir a reação imunológica / inflamatória. De fato, os receptores funcionais de vitamina $\mathrm{D}(\mathrm{VDR})$ são altamente expressos em linfócitos $\mathrm{B}$ e linfócitos $\mathrm{T}$ e principalmente em monócitos / macrófagos, justificando um papel na modulação das respostas imunes inatas e adaptativas (Panfili, Roversi, D'Argenio, et al., 2020).

A vitamina $\mathrm{D}$ é um hormônio esteroidal (hormônio $\mathrm{D}$ ) e pode influenciar a resposta imune em COVID-ı. A vitamina $\mathrm{D}$ entra no corpo através do consumo dietético (cerca de 
$20 \%$ da vitamina $\mathrm{D}_{3}$ ) ou é sintetizada pela pele (80\%) a partir do 7 -di-hidro-colesterol (provitamina D ou clecalciferol) após a exposição ao ultravioleta tipo B (UVB), que varia sazonalmente. A descoberta da presença de VDR em células $\mathrm{T}$ ativadas e monócitos, sugeriu pela primeira vez em 1983 que a vitamina D pode ter um papel na função do sistema imunológico.(Ilie, Stefanescu, Smith, 2020).

De fato, a vitamina $D$ tem recebido cada vez mais atenção mundial por seu envolvimento na redução do risco de várias doenças crônicas, além de doenças infecciosas, incluindo diabetes tipo I e notadamente doenças reumáticas autoimunes, pelo motivo que pode interferir no sistema imunológico.

A evidência biológica / molecular para as interações da vitamina D com a resposta imune é que seu metabólito ativo final, ou seja, o calcitriol $\left(\mathrm{I}, 25(\mathrm{OH})_{2} \mathrm{D}_{3}\right)$, devido à sua origem estrutural do colesterol, é considerado molecularmente um hormônio esteroide (D hormônio) como outros (ou seja, hormônios sexuais, cortisol) e analogamente aos glicocorticoides (e hormônios sexuais) podem exercer atividades imunomoduladoras / antiinflamatórias por meio de receptores de esteroides de células funcionais ( $\mathrm{Li}$ et al, 2020).

Além disso, a intensidade e a qualidade da resposta imune / inflamatória do hospedeiro parecem influenciar a gravidade clínica e o risco de mortalidade associado a doenças virais (como influenza e COVID-Ig) ao invés do próprio patógeno viral (Jakovac, 2020). Consequentemente, é biologicamente plausível que $1,25(\mathrm{OH})_{2} \mathrm{D}_{3}$ possa exercer efeitos imunomoduladores também em pacientes com COVID-I9, desempenhando um papel na regulação da imunidade inata e adaptativa (Bilezikian, Bikle, Hewison, et al., 2020).

A conversão intracelular de $25(\mathrm{OH}) \mathrm{D}$ (calcidiol ou calcifediol) no metabólito ativo I,25 (OH) 2 $\mathrm{D}_{3}$, (calcitriol), através das ações intracrinas da enzima I-alfa-hidroxilase $\left(\mathrm{CYP}_{27} \mathrm{BI}\right)$, é distinta da $1,25(\mathrm{OH})_{2} \mathrm{D}_{3}$ produzido nos rins e liberado na circulação sistêmica; no entanto, ambos têm funções autócrinas e parácrinas que aumentam a imunidade do hospedeiro, por exemplo, regulando positivamente os peptídeos antimicrobianos catelicidina e alfa-defensina (Bilezikian, Bikle, Hewison, et al., 2020). 
Além disso, o epitélio pulmonar também expressa o VDR e o $\mathrm{CYP}_{27} \mathrm{~B}_{1}$ e pode ser um importante tecido-alvo para o sistema endócrino da vitamina D. A imunidade inata é a primeira linha de defesa contra bactérias e vírus e é ativada horas após a exposição ao patógeno (Mathyssen, Aelbrecht, Serré, 2020).

$\mathrm{O}$ calcitriol $\left(\mathrm{I}, 25(\mathrm{OH})_{2} \mathrm{D}_{3}\right)$ pode inibir citocinas de células $\mathrm{T}$ inflamatórias, como a interleucina (IL) -2 e IL-17, e receptores toll-like presentes em monócitos. Por outro lado, em resposta à administração de calcitriol, as células Th em um ambiente inflamatório exibem um potencial aumentado para polarização Th2 juntamente com um potencial diminuído para polarização Thı7. (Chun-Sheng, Li, Hong-Lei et al., 2020).

Altas doses de suplementação de calcitriol em indivíduos humanos saudáveis ( $\mu \mathrm{g}$ duas vezes por dia durante 7 dias) levam a uma redução dramática nos níveis de citocina pró-inflamatória IL-6, secretada por células mononucleares periféricas. Todos esses efeitos provavelmente se combinam e se traduzem na indução de potenciais células $T$ reguladoras, que são importantes para regular as respostas imunes e para o desenvolvimento da auto reatividade (Fisher, Rahimzadeh, Brierley, et al., 2019).

A imunidade adaptativa é o processo imunológico pelo qual a memória imunológica a um antígeno específico é estabelecida e requer um cronograma muito mais lento do que a imunidade inata. A imunidade adaptativa é ainda mediada por dois tipos de células linfocíticas: células $\mathrm{T}$ para imunidade mediada por células e células $\mathrm{B}$ que são responsáveis pela imunidade humoral. As células $B$ ativadas produzem anticorpos específicos para o patógeno e os neutralizam ou destroem por meio de uma variedade de mecanismos.

Foi demonstrado que o calcitriol suprime a imunidade adaptativa em modelos animais, no entanto, dados recentes ainda não são suficientes para provar um papel real da vitamina D na modulação do sistema imunológico adaptativo em humanos (Sassi, Tamone, D'Amelio,. 2018). Por último, a "tempestade de citocinas" é um termo usado para descrever uma resposta imune hiperativa ao COVID-19. Embora ainda haja muito a aprender sobre a fisiopatologia da "tempestade de citocinas", ela é considerada mediada pela ativação do sistema imunológico inato e com uma ativação excessivamente aumentada da imunidade adaptativa (Leisman et al., 2020). 
As manifestações clínicas desta reação aguda incluem lesão pulmonar crítica, dano generalizado ao tecido, falência de múltiplos órgãos e frequentemente morte. Em relação à vitamina $\mathrm{D}$ e ao gênero, sabe-se que a mortalidade é maior em pacientes COVID-Ig do sexo masculino do que do sexo feminino, possivelmente porque, devido aos andrógenos, a resposta imune adaptativa em homens é menos eficiente para montar uma resposta de anticorpos (contra SARS-CoV-2 ), com o resultado de que sua doença evolui ainda mais severamente (Lipsky, Hung, 2020).

Ao contrário, pacientes com COVID-ig do sexo feminino reagem imunologicamente melhor devido ao aumento dos efeitos estrogênicos na imunidade adaptativa, com o resultado de produzir anticorpos específicos que podem neutralizar mais precocemente o vírus, como acontece com outras infecções e após a vacinação (Cutolo, Smith, Paolino, 2020).

Curiosamente, foi relatado que em pacientes COVID-ı9 graves, a média das concentrações séricas de anticorpos IgG SARS-CoV-2 em mulheres tendeu a ser mais alta e a geração de anticorpos IgG foi mais forte em pacientes do sexo feminino do que em pacientes masculinos (Zeng, Dai, Cai, et al., 2020).

Portanto, a deficiência de vitamina $\mathrm{D}$ parece adicionar mais riscos para os pacientes masculinos com COVID-ı; de fato, as concentrações de $25(\mathrm{OH})$ D foram encontradas significativamente mais baixas apenas em pacientes masculinos COVID-19 vs mulheres e controles $(\mathrm{p}=0,0006)$ e não foram confundidos por comorbidades impactadas pela vitamina $\mathrm{D}$ e sazonalidade. Conclusão, a deficiência de vitamina $\mathrm{D}$ parece um fator de risco prevalente e adicional para pacientes com COVID-I9 graves do sexo masculino (La Vignera, Cannarella, Condorelli, et al., 2020).

\section{ICONCLUSÃO}

Dadas as evidências que apoiam o papel da vitamina D na modulação da função imunológica e o impacto da suplementação de vitamina $\mathrm{D}$ em pacientes com deficiência de vitamina D com COVID-ıg, bem como o perfil de segurança favorável (e baixo custo) da vitamina $\mathrm{D}$, recomendações práticas devem ser sintetizadas da forma apresentada a seguir. 
As diretrizes atuais de saúde pública para otimizar o status de vitamina $\mathrm{D}$ devem ser sempre seguidas e os dados clínicos de revisões sistemáticas e metanálises mostram benefícios na prevenção de infecções respiratórias e melhora da função pulmonar quando pacientes com deficiência de vitamina $\mathrm{D}$ são suplementados.

O estado ideal de vitamina $\mathrm{D}$ do hospedeiro pode contribuir com funções imunorreguladoras essenciais em ambientes de infecção respiratória viral e, em geral, a reatividade imune-inflamatória COVID-i9 alterada, pelo menos regulando negativamente as respostas de citocinas excessivamente exuberantes (tempestade de citocinas patológicas, de fato, níveis mais elevados de vitamina $\mathrm{D}$ se correlacionam com níveis mais baixos de IL6).

Pacientes hospitalizados com COVID-19 devem ter as concentrações séricas de 25 $(\mathrm{OH}) \mathrm{D}$ basais medidas e devem ser suplementados a um nível> $30 \mathrm{ng} / \mathrm{mL}$ (ideal 40-6o ng $/ \mathrm{mL}$ ), especialmente quando o nível basal é <io $\mathrm{ng} / \mathrm{mL}$.

Em pacientes COVID-19 com concentrações séricas de $25(\mathrm{OH})$ D abaixo de $20 \mathrm{ng} /$ $\mathrm{mL}$, a dose recomendada para correção da deficiência é de 6.000 a 7.000 UI por via oral por 6 a 8 semanas. Para manutenção, a dose varia de 2.000 a 3.000 UI por via oral / dia dependendo da idade e condição clínica do indivíduo até atingir as concentrações ideais.

Quando não é possível medir as concentrações basais de $25(\mathrm{OH}) \mathrm{D}$ em pacientes com COVID-I9, parece essencial suplementar com 2.000 a 3.000 UI por dia até as concentrações séricas ideais sugeridas (40 a $60 \mathrm{ng} / \mathrm{mL}$ ).

Nos ensaios randomizados e meta-análises, a suplementação de vitamina D demonstrou ter efeitos protetores contra infecções do trato respiratório; portanto, as pessoas com maior risco de deficiência de vitamina D durante esta pandemia global devem considerar a ingestão de suplementos de vitamina $\mathrm{D}$ para manter os níveis ideais de $25(\mathrm{OH})$ Din circulante $\left(75^{-125} \mathrm{nmol} / \mathrm{L}\right)$. 
Alguns estudos retrospectivos demonstraram uma correlação entre a vitamina $\mathrm{D} e$ os casos e desfechos do COVID-ıg, enquanto outros estudos não encontraram a correlação quando as variáveis de confusão são ajustadas.

No entanto, não há evidências suficientes sobre a associação entre os níveis de vitamina $\mathrm{D}$ e a gravidade e mortalidade do COVID-19. Portanto, ensaios clínicos randomizados e estudos de coorte em grande escala são necessários para testar essa hipótese.

\section{REFERÊNCIAS}

ALI N. Elevated level of C-reactive protein may be an early marker to predictrisk for severity of COVID-r9. J Med Virol 2020, jmv.26097.

ALIPIO M. Vitamin D supplementation could possibly improve clinical outcomesof patients infected with coronavirus-2019 (COVID-2019). SSRN3571484; 2020.

BILEZIKIAN JP, BIKLE D, HEWISON M, et al. Mechanisms in endocrinology: vitamin $D$ and COVID-19. Eur J Endocrinol 2020;183:R133-47.doi:10.1530/EJE-20o665pmid:http://www.ncbi.nlm.nih.gov/pubmed/32755992.

BOUILLON R, MARCOCCI C, CARMELIET G, BIKLE D, WHITE JH, DAWSONHUGHES B, et al. Skeletal and extraskeletal actions of vitamin D: current evidence andoutstanding questions. Endocrine Rev 2019;40:1109-51.

CHAROENNGAM N, SHIRVANI A, KALAJIAN TA, SONG A, HOLICK MF. The effect of various doses of oral vitamin $\mathrm{D}_{3}$ supplementation on gut microbiota in healthyadults: a randomized, double-blinded, dose-response study. Anticancer Res 2020;40:551-6.

CHUN-SHENG BI CS, LI X, HONG-LEI QU HL, et al. Calcitriol inhibits osteoclastogenesis in an inflammatory environment by changing the proportion and function of $\mathrm{T}$ helper cells ubsets (Th2/Th17). Cell Prolif 2020;53:e1287.

CUTOLO M, SMITH V, PAOLINO S. Understanding immune effects of oestrogens to explain the reduced morbidity and mortality in female versus male COVID-19 patients. 
Comparisons with autoimmunity and vaccination. Clin Exp Rheumatol 2020;38:3836.pmid:http://www.ncbi.nlm.nih.gov/pubmed/32452350.

DANESHKHAH A, AGRAWAL V, ESHEIN A, SUBRAMANIAN H, ROY HK, BACKMAN $\mathrm{V}$. The possible role of vitamin $\mathrm{D}$ in suppressing cytokine storm and associated mortality in COVID-19 patients [preprint]. Infect Dis (except HIV/AIDS) 2020.

DANKERS W, COLIN EM, VAN HAMBURG JP, LUBBERTS E. Vitamin D in autoimmunity: molecular mechanisms and therapeutic potential. Front Immunol 2017;7:697.

DARLING AL, AHMADI KR, WARD KA, HARVEY NC, COUTO ALVES A, DUNN-WATERS DK, et al. Vitamin D status, body mass index, ethnicity and COVID19: Initial analysis of the first-reported UK Biobank COVID-19 positive cases (n 580) comparedwith negative controls ( $\mathrm{n}$ 723) [preprint]. Infect Dis (except HIV/AIDS) 2020.

DE SMET D, DE SMET K, HERROELEN P, GRYSPEERDT S, MARTENS GA.

Vitamin D defi-ciency as risk factor for severe COVID-19: a convergence of two pandemics [preprint]. Infect Dis (except HIV/AIDS) 2020.

FABBRI A, INFANTE M, RICORDI C. Editorial-Vitamin D status: a key modulator ofinnate immunity and natural defense from acute viral respiratory infections. Eur Rev Med Pharmacol Sci 2020;24:4038-42.

FISHER SA, RAHIMZADEH M, BRIERLEY C, et al. The role of vitamin D in increasing circulating $\mathrm{T}$ regulatory cell numbers and modulating $\mathrm{T}$ regulatory cell phenotypes in patients with inflammatory disease or in healthy volunteers: a systematic review. PLoS One 2019;14:e0222313. doi:Io.137I/journal.pone.0222313pmid:http://www.ncbi.nlm.nih.gov/pubmed/31550254.

GLICIO EJ. Vitamin D level of mild and severe elderly cases of COVID-rg: a preliminary report. SSRN: https://ssrn.com/abstract=3593258; 2020 . 
GOMBART AF, PIERRE A, MAGGINI S. A review of micronutrients and theimmune system-working in harmony to reduce the risk of infection. Nutrients 2020;12:236.

GRANT WB, AL ANOUTI F, MOUKAYED M. Targeted 25-hydroxyvitamin D concentration measurements and vitamin $\mathrm{D}_{3}$ supplementation can have important patient and public health benefits. Eur J Clin Nutr 2020;74:366-76. doi:10.1038/s41430-020-0564opmid:http://www.ncbi.nlm.nih.gov/pubmed/31996793.

GRANT WB, LAHORE H, MCDONNELL SL, BAGGERLY CA, FRENCH CB, ALIANO JL, et al. Evidence that vitamin D supplementation could reduce risk of influenza andCOVID-19 infections and deaths. Nutrients 2020;12:988.

GUAN W, LIANG W, ZHAO Y, LIANG H, CHEN Z, LI Y, et al. Comorbidity and its impacton 1590 patients with COVID-19 in China: a nationwide analysis. Eur Respir J2020;55:2000547.

HASTIE CE, MACKAY DF, HO F, CELIS-MORALES CA, KATIKIREDDI SV, NIEDZWIEDZ CL, et al. Vitamin D concentrations and COVID-19 infection in UK Biobank. DiabetMetab Syndr: Clin Res Rev 2020;14:56I-5.

HOLICK MF. The vitamin D deficiency pandemic: approaches for diagnosis, treatment and prevention. Rev Endocrine Metab Disord 2017;18:153-65.

HOU Y-M, ZHAO J-Y, LIU H-Y. Impact of serum 25-hydroxyvitamin $\mathrm{D}$ on cardiacprognosis in Chinese patients with heart failure. Br J Nutr 2019;122:162-71.

ILIE PC, STEFANESCU S, SMITH L. The role of vitamin D in the prevention of coronavirus disease 2019 infection and mortality. Aging Clin Exp Res 2020;32:1195-8.

JAKOVAC H. COVID- $\mathrm{H} 9$ and vitamin $\mathrm{D}$-is there a link and an opportunity forintervention? Am J Physiol Endocrinol Metab 2020;318:E589.

JI W, HUH K, KANG M, HONG J, BAE GH, LEE R, et al. Effect of underlying comorbidities on the infection and severity of COVID-19 in South Korea [preprint]. InfectDis (except HIV/AIDS) 2020, http://dx.doi.org/ro.I10I/2020.05.08.20095174. 
LA VIGNERA S, CANNARELLA R, CONDORELLI RA, et al. Sex-Specific SARSCoV-2 mortality: among Hormone-Modulated $\mathrm{ACE}_{2}$ expression, risk of venous thromboembolism and hypovitaminosis D. Int J Mol Sci 2020;21:ijms21082948. doi:10.3390/ijms21082948pmid:http://www.ncbi.nlm.nih.gov/pubmed/32331343PubMedGo ogle Scholar

LEI G-S, ZHANG C, CHENG B-H, LEE C-H. Mechanisms of action of vitamin Das supplemental therapy for pneumocystis pneumonia. Antimicrob AgentsChemother 2017;6r.

LEISMAN DE, RONNER L, PINOTTI R, et al. Cytokine elevation in severe and critical COVID-19: a rapid systematic review, meta-analysis, and comparison with other inflammatory syndromes. Lancet Respir Med 2020;8:1233-44.

LI Y, LI Q, ZHANG N, LIU Z. Sunlight and vitamin D in the prevention of coronavirusdisease (COVID-19) infection and mortality in the United States (preprint, inreview); 2020. doi:10.21203/rs.3.rs-32499/vi.

LIPS P, CASHMAN KD, LAMBERG-ALLARDT C, BISCHOFF-FERRARI HA, OBERMAYER-PIETSCH B, BIANCHI ML, et al. Current vitamin D status in European and MiddleEast countries and strategies to prevent vitamin D deficiency: a position statement of the European Calcified Tissue Society. Eur J Endocrinol 2019:P23-54.

LIPSKY MS, HUNG M. Men and COVID-rg: a pathophysiologic review. Am J Mens Health 2020;14:1557988320954021. doi:10.1177/1557988320954021pmid:http://www.ncbi.nlm.nih.gov/pubmed/32936693

MARTINEAU AR, JOLLIFFE DA, HOOPER RL, et al. Vitamin D supplementation to prevent acute respiratory tract infections: systematic review and meta-analysis of $\begin{array}{lll}\text { individual participant data } & \text { 2017;356:16583. }\end{array}$ doi:10.1136/bmj.i6583pmid:http://www.ncbi.nlm.nih.gov/pubmed/28202713

MATHYSSEN C, AELBRECHT C, SERRÉ J, et al. Local expression profiles of vitamin D-related genes in airways of COPD patients. Respir Res 2020;21:137. 
MCCARTNEY DM, BYRNE DG. Optimisation of vitamin D status for enhancedimmuno-protection against Covid-19. Irish Med J 2020;113:58.

MOUSAVI S, BERESWILL S, HEIMESAAT MM. Immunomodulatory and antimicrobial effects of vitamin C. Eur J Microbiol Immunol (Bp) 2019;9:73-9.

MUNSHI R, HUSSEIN MH, TORAIH EA, et al. Vitamin D insufficiency as a potential culprit in critical COVID-19 patients. J Med Virol 2020:jmv.26360. doi:10.1002/jmv.2636opmid:http://www.ncbi.nlm.nih.gov/pubmed/32716073.

PANFILI FM, ROVERSI M, D'ARGENIO P, et al. Possible role of vitamin D in Covid19 infection in pediatric population. J Endocrinol Invest 2020:1-9.

PITTAS AG, DAWSON-HUGHES B, SHEEHAN P, WARE JH, KNOWLER WC, ARODA VR, et al. Vitamin D supplementation and prevention of type 2 diabetes. $\mathrm{N}$ Engl J Med2019;381:520-30.

RAHARUSUN P, PRIAMBADA S, BUDIARTI C, AGUNG E, BUDI C. Patterns of 1079 COVID-19 mortality and vitamin D: an Indonesian study. SSRN J 2020.

RHODES JM, SUBRAMANIAN S, LAIRD E, et al. Perspective: vitamin D deficiency and COVID-19 severity - plausibly linked by latitude, ethnicity, impacts on cytokines, ACE2 and thrombosis. J Intern Med 2020;II3.doi:I0.IIII/joim.I3149Google Scholar

SASSI F, TAMONE C, D'AMELIO P. Vitamin D: nutrient, hormone, and immunomodulator. Nutrients 2018;10:1656. doi:10.3390/nurorir656pmid:http://www.ncbi.nlm.nih.gov/pubmed/30400332

SURVEILLANCES V. The epidemiological characteristics of an outbreak of 2019 novel coronavirus diseases (COVID-19) - China, 2020. China CDC Wkly2020;2:113-22.

TAN CW, HO LP, KALIMUDDIN S, CHERNG BPZ, TEH YE, THIEN SY, et al. A cohortstudy to evaluate the effect of combination Vitamin D. Magnesium and Vitamin B12 (DMB) on progression to severe outcome in older COVID-i9 patients [preprint]. Infect Dis (except HIV/AIDS) 2020, http://dx.doi.org/ro.110I/2020.06.01.20112334. 
TEYMOORI-RAD M, SHOKRI F, SALIMI V, MARASHI SM. The interplay between vitamin D and viral infections. Rev Med Virol 2019;29:e2032.

TIAN Y, RONG L. Covid-rg and vitamin D-authors' reply. Aliment Pharmacol Ther 2020. TSUJINO I, USHIKOSHI-NAKAYAMA R, YAMAZAKI T, MATSUMOTO N, SAITO I. Pulmonaryactivation of vitamin $\mathrm{D}_{3}$ and preventive effect against interstitial pneumonia. J Clin Biochem Nutr 2019;65:245-51.

UWITONZE AM, RAZZAQUE MS. Role of magnesium in vitamin D activation and function. J Am Osteopath Assoc 2018;118:18I-9.

VANKADARI N, WILCE JA. Emerging WuHan (COVID-rg) coronavirus: glycan shield and structure prediction of spike glycoprotein and its interaction with humanCD26. Emerg Microb Infect 2020;9:60I-4.

VYAS N, KURIAN SJ, BAGCHI D, et al. Vitamin D in prevention and treatment of COVID-19: current perspective and future prospects. J Am Coll Nutr 2020;1:1I4.doi:I0.1080/o7315724.2020.1806758pmid:http://www.ncbi.nlm.nih.gov/pubmed/32870735.

YANG J, ZHENG Y, GOU X, PU K, CHEN Z, GUO Q et al. Prevalence of comorbiditiesand its effects in patients infected with SARS-CoV-2: a systematic review and meta-analysis. Int J Infect Dis 2020;94:91-5.

ZDRENGHEA MT, MAKRINIOTI H, BAGACEAN C, BUSH A, JOHNSTON SL, STANCIU LA. Vitamin D modulation of innate immune responses to respiratory viral infections. Rev Med Virol 2017;27:e1909.

ZENG F, DAI C, CAI P, et al. A comparison study of SARS-CoV-2 IgG antibody between male and female COVID-rg patients: a possible reason underlying different outcome between sex. J Med Virol 2020;92:20504.doi:Io.1002/jmv.25989pmid:http://www.ncbi.nlm.nih.gov/pubmed/32383183PubMedGoo gle Scholar ZHOU Y-F, LUO B-A, QIN L-L. The association between vitamin D deficiency andcommunity-acquired pneumonia: a meta-analysis of observational studies. Medicine 2019;98. 


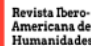

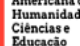

Revista Ibero- Americana de Humanidades, Ciências e Educação- REASE

open 2 access 